\title{
Académie des sciences
}

\section{Rapport Science et Technologie}

Le Comité interministériel du 15 juillet 1998, à l'initiative du ministre de l'Éducation nationale, de la Recherche et de la Technologie, a confié à l'Académie des sciences l'établissement du rapport biennal sur l'état de la science et de la technologie.

Pour répondre à cette demande, l'Académie des sciences a mis en place le Comité "Rapport Science et Technologie » (RST) constitué de 15 membres de la Compagnie et de son Conseil pour les applications.

Chaque thème retenu est conduit par un groupe de travail animé par un membre ou un correspondant de l'Académie, entouré d'experts.

Chaque rapport est soumis au Comité RST, à un Groupe de lecture critique, et à l'Académie des sciences.

En 2001, la composition du Comité RST a été élargie, passant de quinze à vingt-quatre membres.

Entre 1999 et 2005, vingt rapports ont ainsi été édités et remis au ministre délégué à la Recherche. 
